\title{
Cardio-oncology in Ukraine: experience at Strazhesko Institute of Cardiology
}

\section{Sergey Kozhukhov' , Nataliia Dovganych', Ivan Smolanka', Olga Lyhyrda}

${ }^{1}$ Cardio-Oncology Unit, National Scientific Center M.D. Strazhesko Institute of Cardiology, Kyiv, Ukraine

${ }^{2}$ Ukrainian National Cancer Institute, Kyiv, Ukraine

Correspondence:

Sergey Kozhukhov

Department of Clinical Pharmacology, Cardio-Oncology Unit, National Scientific Center M.D. Strazhesko Institute

of Cardiology, Kiev, Ukraine e-mail:s.kozhukhov@i.ua

Received: 13.08.2018 Accepted: 12.09.2018

DOI: 10.24292/01.OR.120918 Copyright @ Medical Education. All rights reserved.

\section{ABSTRACT}

Anticancer therapies have extended the lives of patients with cancer, but for some, this benefit is tempered by cardiovascular complications. Their number is increasing as a result of an improvement in the early diagnosis of cardiotoxicity caused by chemo- and radiotherapy. Therefore prevention, detection, monitoring, and treatment of cancer patients at risk of cardiotoxicity with or without concomitant CV diseases are very important. Cardio-oncology is a new direction in Ukraine for improving clinical outcomes of cancer patients. This review aims to provide an overview of the rationale for setting up a Cardio-Oncology Unit and reflects our own experience establishing this service.

Key words: cardio-oncology, cardiotoxicity, cardiovascular risk factors 


\section{BACKGROUND}

During the last decades number of patients with cardiovascular complications caused by anti-tumor therapy has increased as a result of improved early diagnosis of chemotherapy- and radiotherapy-induced cardiotoxicity along with population growth and aging [1-3]. Besides cardiac dysfunction/heart failure, cardiotoxicity is presented by arterial hypertension, ischemia, arrhythmias and thromboembolic complications, which can negatively impact outcomes of cancer patients $[4,5]$.

According to the Ukrainian State Expert Center data impact of reported adverse reactions due to anticancer drugs is not very high $(2,5 \%)$ due to low reporting activity. At the same time, cardiac complications range from 11 to $18 \%$ and are presented mainly by heart failure, increased blood pressure and rhythm disturbances. Also it should be noted that there is an increase in the adverse reactions reporting from $26 \%$ to $39 \%$ during the last 5 years [6].

Therefore, early detection of cancer therapy-related toxicity has become a clinical concern for oncologists, hematologists, and cardiologists $[7,8]$. An integrative multidisciplinary approach contributed to the emergence of new subspeciality - cardio-oncology $[9,10]$. Distinguishing tumor-associated or iatrogenic cardiotoxicity from inherent cardiac diseases is one of the tasks of cardio-oncology. In recent years, the number of cardio-oncology units has rapidly increased and become more of a formal subspecialty with smaller units or services within major centers in European countries, Canada and USA.

The main purpose of cardio-oncology includes prevention, detection, monitoring and treatment of cardiovascular toxicity related to cancer therapy in order to improve quality of life and survival of cancer patients.

\section{CARDIOTOXICITY}

The main cardiovascular complications of cancer therapy are myocardial dysfunction and heart failure, coronary artery disease, valvular disease, arrhythmias, arterial hypertension, thromboembolic disease, peripheral vascular disease, pulmonary hypertension and pericardial complications.

The cardiotoxicities of chemotherapy and radiation therapy can be broadly classified as acute or late effects. The acute effects of cancer treatment primarily impact the vascular system, specifically leading to hypertension, vasospasm, arterial or venous thrombosis and myocarditis.
Two types of cardiotoxicity have been described - a dose-dependent, cumulative, mostly irreversible form (anthracyclines) and a reversible, dose-independent type (trastuzumab).

Late effects are long-term toxicities that generally involve structural cardiovascular changes, including atherosclerosis, valvular heart disease, and conduction system disease.

Finally, newer treatment modalities such as immune checkpoint inhibition can cause systemic inflammation and cardiac toxicity, including end-organ myofibrosis, autoimmune myocarditis, and acute heart failure [11, 12].

\section{BACKGROUND FOR A MULTIDISCIPLINARY APPROACH}

Data from registries and randomized clinical trials show that cardiovascular complications from cancer therapy have become a leading cause of morbidity and mortality in cancer survivors [13]. Anticancer therapies have improved survival and prolonged the lives of patients with cancer, but for some at the cost of adverse cardiovascular events [14]. Comorbidities, pre-existing heart diseases and age are the most contributing factors in the onset of cardiotoxicity.

Therefore the multidisciplinary integrative approach of cardio-oncology is needed $[15,16]$. In this case, cardio-oncology aims not only to detect and manage cardiotoxicity, but also to assist the overall care of cancer patients in an interdisciplinary manner. The main goal of the oncologist is to provide cancer treatment in accordance with protocols and terms with the aim of curing or prolonging the life of cancer patient [17].

Cardio-oncology's task is to evaluate oncological and cardiovascular risks, to recognize patients, who are at increased risk for developing cardiac dysfunction, to treat comorbidities, to solve the problem how anti-tumor therapies can be given safely to patients with malignancies before and during cancer treatment. Mutual understanding and communication between the cardiologist and oncologist are needed in a process of cancer therapy and in case of cardiovascular complications occurrence. Accordingly, one of the main goals of cardio-oncology is to promote open discussion between team members in order to share experience and responsibilities to provide a high-level standard of care [18]. 


\section{SETTING UP THE CARDIO-ONCOLOGY PRACTICE} IN UKRAINE

Cardio-oncology Unit was formed at the Clinical Pharmacology Department of Strazhesko Institute of Cardiology in 2016 in order to improve the short- and long-term outcomes of cancer patients. This subspecialty was initially created:

- to diagnose, manage and monitoring cardiovascular complications of cancer therapy

- to evaluate baseline cardiovascular risks prior to anti-tumor treatment and implement strategies for risk reduction of developing cardiovascular complications during cancer treatment

- to assist the oncological patient with cardiovascular care through long-term follow-up.

In USA, Canada and European countries cardio-oncology centers mainly located in central cancer clinics or multidisciplinary hospitals.

Cardio-Oncology Unit of Strazhesko Institute of Cardiology is unique by being first in Ukraine and due to rendering much more assistance to oncological patients as the main cardiological clinic of the country. The multidisciplinary team in Strazhesko Institute of Cardiology consists of cardiologists with additional experience in heart failure, arrhythmias with possibility of implanting devices, catheterization lab, emergency care and cardiovascular imaging.

In Strazhesko Cardio-Oncology Unit patients get diagnostic and treatment support and can be redirected to special procedures which they need.

The cardio-oncology practice in Strazhesko Institute of Cardiology was initially established through the outpatients consulting. Face-to-face consultations emerged as a mechanism to provide effective clinical care in a timely manner.

A formal Strazhesko Cardio-Oncology Unit was then started with inpatient treatment and had a significant growth over the past 2 years.

The monthly number of visits has increased by $50 \%$ since 2017 to 2018 , monthly consulting average $15-20$ new patients (fig. 1). The ratio between new and old patients is $2: 1$. Breast cancer was the most frequent cancer type (55\%), followed by hematological malignancies (16\%), lung cancer (10\%) and others 19\% (renal metastatic cancer etc (fig. 2).
FIGURE 1.

Cardio-Oncology Unit visits.

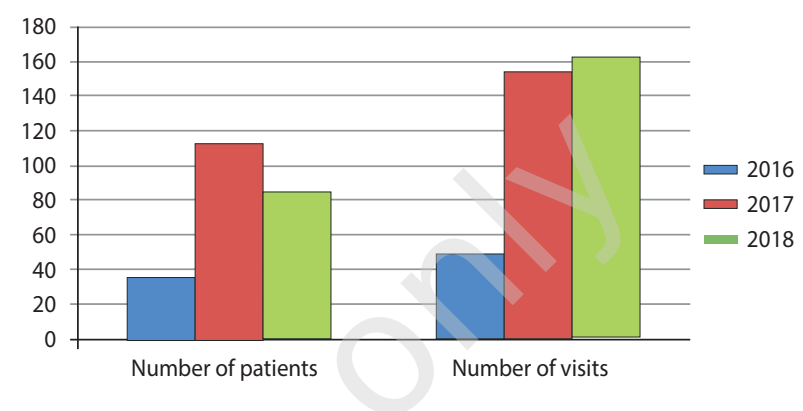

FIGURE 2.

Distribution of patients according to the diagnosis.

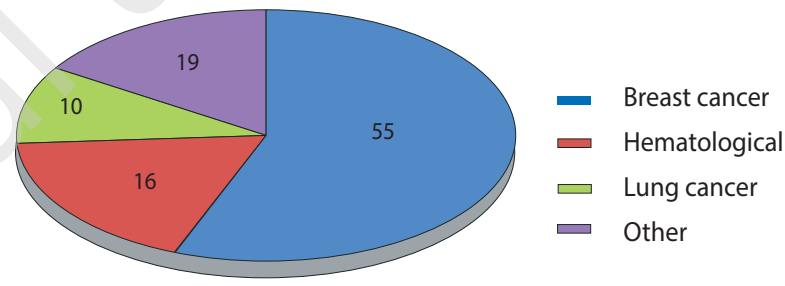

The most common reasons for referral to the Cardio-Oncology Unit were decreased LVEF (72, 19.7\%), pre-chemotherapy assessment $(84,23.0 \%)$, and symptomatic heart failure $(39,10.7 \%)$, hypertension $(38,10.4 \%)$. Less common reasons included palpitations, arrhythmia and cardiomyopathy (tab. 1).

TABLE 1.

Reasons for Referral to Cardio-Oncology Unit.

\begin{tabular}{|c|c|}
\hline & N of visits = 365 \\
\hline Reason & $\mathrm{N}(\%)$ \\
\hline Decreased LVEF & $72(19.7 \%)$ \\
\hline Pre-chemotheraphy assessment & $84(23.0 \%)$ \\
\hline Symptomatic heart failure & $39(10.7 \%)$ \\
\hline $\begin{array}{c}\text { Hypertension/blood pressure } \\
\text { increase }\end{array}$ & $38(10.4 \%)$ \\
\hline Palpitations & $34(9.3 \%)$ \\
\hline Arrhythmia & $18(4.9 \%)$ \\
\hline Cardiomyopathy & $15(4.1 \%)$ \\
\hline Other & $65(17.9 \%)$ \\
\hline
\end{tabular}

Cancer drugs that are currently in use and novel agents with target signaling pathways may all cause heart problems. As the complexity of cancer treatments has increased, so have the tools available to monitor the impact on the heart during anti-tumor therapy and long after it. Currently $80 \%$ of patients consulted in Strazhesko Cardio-Oncology Unit received anthracyclines. During last year we have managed and monitored the breast cancer patients treated 
with anti-HER2 drugs, which have a positive anticancer effect but can lead to LV ejection fraction decline and heart failure.

The patients with malignancies and cardiovascular complications need complex care, which requires not only medical specialists, but also medical assistants, nurses, nurse practitioners and physician extenders (physician assistants, fellows and internists). Some of these patients are in the intermediate or high-risk category for coronary artery disease, cerebrovascular disease or heart failure. Their management also includes coordinated home health monitoring of blood pressure, cholesterol levels, diuresis, medications adjustments, and evaluation of new symptoms through e-mail or phone calls. Primary physicians are able to treat more stable or returning patients, therefore allowing the cardio-oncologist manage mostly the patients with severe complications.

The goals of Strazhesko Cardio-Oncology Unit during this year are as follows:

- to organize a Cardio-Oncology session at the Ukrainian Congress of Cardiologists

- to prepare, edit and implement the standards/protocols for management the cancer patients with cardiovascular complications

- to observe breast cancer patients included in ESC-COT (Cardiac-Oncology Toxicity) EACVI/HFA Pilot registry [19]

- to expanse of care increasing referral and a quantity of patients as well as further integration into survivorship and rehabilitation

- to join educational sessions in the field of oncology, hematology and radiation therapy

- to apply for institutional and international grants

- to initiate a new pilot clinical studies

- to establish experimental collaborations and registry expansion.

We hope that Strazhesko's Cardio-Oncology Program may bring improvement in clinical outcomes and contribute to health and well-being in cancer patients.

\section{BASELINE AND MONITORING EVALUATION OF CANCER PATIENTS}

Predicting the risk of cardiotoxicity, both from a clinical and a practical point of view has a very high priority, as it allows better distribution and individualization of therapy.

As per 2016 ESC Position Paper on Cancer Treatments and Cardiovascular Toxicity recommendations has been recently proposed from the ESC Committee for Practice Guidelines, where cancer therapeutics-related cardiac dysfunction is defined as a decrease in LVEF of $>10 \%$, to a value $<50 \%$ (lower limit) [7].

Strazhesko Cardio-Oncology Unit has established a standardized approach based on ESC position paper and own experience gained in leading European clinics. All patients with potential risk of cardiotoxicity undergo a comprehensive echocardiographic evaluation and biomarkers screening at baseline, during therapy and follow-up, which depends on the type of anti-tumor agent and its combination with radiation.

Follow-up monitoring varies according to the risk factors and individual patient's characteristics and/or genetic susceptibility. For patients at increased cardiac risk more aggressive cardiac monitoring is recommended.

Strazhesko cardio-oncology consultation's algorithm includes detailed medical history, type of planned anticancer therapy and the presence of risk factors. As an important part of baseline clinical work-up prior to cancer treatment ECG, biomarkers (hsTn, brain natriuretic peptide in selected patients), and echocardiography imaging are recommended in our unit for all patients who need to undergo treatment with potential risk of cardiotoxicity.

In our opinion, in order to achieve the best results in treatment of oncological patients, a team collaboration of the cardiologist and oncologist, a systematic approach to the organization of cardio-oncology services, the use of advanced technologies and the exchange of experience are needed.

\section{CONCLUSION}

Cardio-oncology is a growing field that aims to optimize the cardiovascular care of cancer patients before, during and after chemotherapy and/or radiation therapy.

Cardio-oncology is an important sub-specialization at the intersection of oncology and cardiology. Indeed, cardiovascular diseases can significantly increase morbidity in cancer survivors. The cardio-oncological team plays an important role in the management of oncological patients to decrease cardiovascular morbidity by early risk factor modification, serial monitoring with imaging and biomarkers, optimal medical therapy for cardiotoxicity when it occurs.

This multidisciplinary partnership is a key element in improving the quality of care for cancer patients. 


\section{References}

1. Cardinale D, Colombo A, Lamantia G et al. Anthracycline-induced cardiomyopathy: clinical relevance and response to pharmacologic therapy. J Am Coll Cardiol 2010; 55(3): 213-220.

2. Cardinale D, Biasillo G, Cipolla CM. Curing cancer, saving the heart: a challenge that cardioncology should not miss. Curr Cardiol Rep 2016; 18 : 51 .

3. Tromp J, Steggink LC, Van Veldhuisen DJ et al. Cardio-oncology: progress in diagnosis and treatment of cardiac dysfunction. Clin Pharmacol Ther 2017; 101: 481-490.

4. Kim KW, Shinagare AB, Krajewski KM et al. Fluid retention associated with imatinib treatment in patients with gastrointestinal stromal tumor: quantitative radiologic assessment and implications for management. Korean J Radiol 2015; 16(2): 304-313.

5. Ewer MS, Ewer SM. Cardiotoxicity of anticancer treatments. Nat Rev Cardiol 2015; 12(9): 547-558.

6. Reports of State Expert Centre of the Ministry of Health of Ukraine [online: www.dec.gov.ua].

7. Zamorano JL, Lancellotti P, Rodriguez Muñoz D et al. 2016 ESC Position Paper cancer treatments and cardiovascular toxicity developed under the auspices of the ESC Committee for Practice Guidelines. Eur Heart J 2016; 37: 2768-2801.

8. Plana JC, Galderisi M, Barac A et al. Expert consensus for multimodality imaging evaluation of adult patients during and after cancer therapy: a report from the American Society of Echocardiography and the European Association of Cardiovascular Imaging. J Am Soc Echocardiogr 2014; 27(9): 911-939.

9. Herrmann J, Lerman A. An update on cardio-oncology. Trends Cardiovasc Med 2014; 24(7): 285-295.

10. Lancellotti P, Suter TM, López-Fernández T et al. Cardio-Oncology Services: rationale, organization and implementation. A report from the ESC Cardio-Oncology council. Eur Heart J 2018; 6. DOI: 10.1093/eurheartj/ehy453 [Epub ahead of print].

11. Läubli $\mathrm{H}$, Balmelli C, Bossard M et al. Acute heart failure due to autoimmune myocarditis under pembrolizumab treatment for metastatic melanoma. J Immunother Cancer 2015; 3: 11.

12. Koelzer VH, Rothschild SI, Zihler D et al. Systemic inflammation in a melanoma patient treated with immune checkpoint inhibitors-an autopsy study. J Immunother Cancer 2016; 4: 13.

13. Barac A, Murtagh G, Carver JR et al. Cardiovascular health of patients with cancer and cancer survivors: a roadmap to the next level. J Am Coll Cardiol 2015; 65: 2739-2746.

14. der Meer P, Gietema JA, Suter TM et al. Cardiotoxicity of breast cancer treatment: no easy solution for an important long-term problem. Eur Heart J 2016; 37: 1681-1683.

15. Nhola LF, Villarraga HR. Rationale for Cardio-Oncology Units. Rev Esp Cardiol 2017; 70: 583-589.

16. Parent S, Pituskin E, Paterson DI. The cardio-oncology program: a multidisciplinary approach to the care of cancer patients with cardiovascular disease. Can J Cardiol 2016; 32: 847-851.

17. Borras JM, Albreht T, Audisio R et al. European Policy statement on multidisciplinary cancer care. European Partnership Action Against Cancer: consensus group. J Cancer 2014; 50: 475-480.

18. Cubbon RM, Lyon AR. Cardio-oncology: concepts and practice. Indian Heart J 2016; 68(Suppl 1): S770-S785.

19. Lancellotti P, Anker SD, Donal E et al. EACVI/HFA Cardiac Oncology Toxicity Registry in breast cancer patients: rationale, study design, and methodology (EACVI/HFA COT Registry) - EURObservational Research Program of the European Society of Cardiology. Eur Heart J Cardiovasc Imaging 2015; 16: 466-470.

Authors' contributions:

Sergey Kozhukov and Nataliia Dovganych conceived of the presented idea. Ivan Smolanka and Olga Lyhyrda performed the statistical analyses for the suggested study. Sergey Kozhukov, Nataliia Dovganych, Ivan Smolanka and Olga Lyhyrda contributed to the design and implementation of the research, to the analysis of the results and to the writing of the manuscript.

Conflict of interests:

None.

Financial support:

None.

Ethics:

The paper complies with the Helsinki Declaration, EU Directives and harmonized requirements for biomedical journals. 